\title{
Induced neural progenitor cells and iPS-neurons from major depressive disorder patients show
} altered bioenergetics and electrophysiological properties

Julian Triebelhorn ${ }^{1 *}$, Kerstin Kuffner ${ }^{1^{*}}$, Iseline Cardon ${ }^{1}$, Katrin Meindl ${ }^{1}$, Tanja Rothhammer-Hampl ${ }^{2}$, Markus J. Riemenschneider ${ }^{2}$, Konstantin Drexler ${ }^{3}$, Mark Berneburg ${ }^{3}$, Caroline Nothdurfter ${ }^{1}$, André Manook $^{1}$, Christoph Brochhausen ${ }^{4,5}$, Thomas C. Baghai ${ }^{1}$, Rainer Rupprecht ${ }^{1}$, Vladimir M. Milenkovic $^{1}$, Christian H. Wetzel ${ }^{1 \S}$

${ }^{1}$ Department of Psychiatry and Psychotherapy, University of Regensburg, Regensburg, Germany

${ }^{2}$ Department of Neuropathology, Regensburg University Hospital, Regensburg, Germany

${ }^{3}$ Department of Dermatology, Regensburg University Hospital, Regensburg, Germany

${ }^{4}$ Institute of Pathology, University of Regensburg, Regensburg, Germany

5 Central Biobank of the University of Regensburg and the Regensburg University Hospital, Regensburg, Germany

\footnotetext{
* These authors contributed equally to this work

${ }^{\S}$ Corresponding author: christian.wetzel@klinik.uni-regensburg.de

Department of Psychiatry and Psychotherapy, University of Regensburg, Universitätsstr. 84, 93053 Regensburg, Germany
}

Keywords: major depression; mitochondria; induced pluripotent stem cells; neural progenitor cells; neurons; OXPHOS; bioenergetics; live cell imaging; electrophysiology 


\section{ABSTRACT}

BACKGROUND: The molecular pathomechanisms of major depressive disorder (MDD) are still not completely understood. Here, we follow the hypothesis, that mitochondrial dysfunction which is inevitably associated with bioenergetic misbalance is a risk factor that contributes to the susceptibility of an individual to develop MDD. MDD can be regarded as disease of the mind as well as of the body. Thus, we focused on mitochondrial and cellular function in reprogrammed neural cells from MDD patients and healthy controls.

METHODS: We investigated molecular mechanisms related to mitochondrial and cellular functions in induced neuronal progenitor cells (NPCs) as well as in neurons, which were reprogrammed from fibroblasts of depressed patients and non-depressed controls, respectively.

RESULTS: We found significantly lower basal respiration rates and a less hyperpolarized mitochondrial membrane potential in NPCs derived from MDD patients. These findings are in line with our earlier observations in patient-derived fibroblasts (1). Furthermore, we differentiated MDD and control NPCs into iPS-neurons and analyzed their passive biophysical and active electrophysiological properties. Interestingly, MDD patient-derived iPS-neurons showed significantly lower membrane capacitance, a more depolarized membrane potential, and increased spontaneous electrical activity. This is the first report showing functional differences in MDD patient-derived and control NPCs and iPS-neurons.

CONCLUSION: Our findings indicate that functional differences evident in fibroblasts from depressed patients are also present after reprogramming and differentiation to induced-NPCs and iPSneurons and might be associated with the aetiology of major depressive disorder. 


\section{INTRODUCTION}

Depressive syndrome is a debilitating and severe mental disorder, affecting about 5 million people per year in Germany and 350 million people worldwide (World Health Organization, 2016). The molecular mechanisms underlying the cause and progress of this complex disease are still not completely understood. Hypotheses argue for a combination of neurobiological factors which affect cellular function and neuronal communication, thereby increasing the risk for MDD, in conjunction with environmental (psychosocial) stress, shown to be associated with the onset and severity of depressive episodes (2). Involved molecular mechanisms include genetically inherited and/or acquired neurobiological issues such as the dysregulation of monoaminergic, glutamatergic and/or GABAergic neurotransmission as well as reduced neuroplasticity and neurogenesis as a consequence of impaired BDNF signaling (reviewed in (3)). Furthermore, mitochondrial dysfunction associated with reduced bioenergetic capability is considered an important risk factor for major depressive disorder as well as other psychiatric disorders (4-6), by constraining proper cellular function and rendering the cells vulnerable to stress, especially during increased metabolic demand. Especially brain neurons rely heavily on mitochondrial energy provision to maintain membrane excitability and operate neurotransmission, as well as to sustain plasticity (7). However, MDD is increasingly viewed as an illness of the mind as well as the body $(8,9)$. Literature already reports mitochondria-related effects in fibroblasts $(1,9)$, muscle- (10), peripheral mononuclear blood cells (11), and platelets $(12,13)$ of depressed patients, suggesting that mitochondria-related pathomechanisms associated with MDD can be identified and studied in neuronal and also in peripheral non-neuronal cells.

To challenge the hypothesis that mitochondria dysfunction contributes to the aetiology of the disease, we aimed to identify and characterize molecular pathomechanisms related to mitochondrial dysfunction and bioenergetic imbalance in a human cellular model of major depression. We used skin fibroblasts from MDD patients and non-depressed controls (1) to reprogram and differentiate these cell lines to neural progenitor cells (NPCs) and neurons (iPS-neurons), shifting our cellular model from peripheral non-neuronal to neuronal cells. The advent of induced pluripotent stem cell (iPSC) technology $(14,15)$ opens a new avenue for studying basic biological and pathological mechanisms $(16,17)$. The generation of disease- and/or patient-specific iPSCs and their differentiation into specific 
cell types helps unravelling the molecular pathomechanisms which underly the etiology of complex diseases such as MDD. As a measure of mitochondrial function/dysfunction we measured the ATPcontent, the mitochondrial membrane potential (MMP), the mitochondrial oxidative phosphorylation system (OXPHOS), and the cytosolic $\mathrm{Ca}^{2+}$ homeostasis in NPCs of depressed patients (MDD) and compared these parameters to non-depressed controls. Interestingly, the reprogrammed NPCs presented functional alterations, which might be associated with bioenergetic dysfunction of mitochondria in MDD patient cells and were in line with findings obtained in the founder fibroblasts (1).

Moreover, in order to characterize electrophysiological properties of neurons derived from depressed patients and to identify molecular phenotypes of mitochondrial impairment in these cells, we allowed the NPCs to differentiate into iPS-neurons. iPS-neurons were analyzed by whole-cell voltage- and current-clamp recording. We found differences in the membrane capacitance, resting membrane potential and spontaneous activity of MDD iPS-neurons when compared with cells derived from the non-depressed controls. In summary, we identified alterations in bioenergetic parameters in NPCs, and found altered electrophysiological properties in iPS-neurons of depressed patients. Moreover, our findings indicate that functional differences evident in fibroblasts from depressed patients (1) are also present after reprogramming and differentiation to induced-NPCs and iPS-neurons and might be associated with the aetiology of major depressive disorder. 


\section{METHODS AND MATERIALS}

Human primary skin fibroblasts derived from 8 depressed patients and 8 non-depressed controls (1) were reprogrammed to iPSCs and differentiated to NPCs using established media, supplements and protocols from STEMCELL Technologies and Life Technologies (see SUPPLEMENTAL METHODS AND MATERIALS). Pluripotency of the iPSCs was analyzed using PluriTest ${ }^{\circledR}$ method (18). To assess mitochondrial function, we measured the oxygen consumption rate (OCR, using Seahorse technology; Agilent Technologies), the cellular ATP content (CellTiter-Glo®Cell Viability Kit; Promega), the mitochondrial membrane potential (JC-1; Life Technologies), and cytosolic $\mathrm{Ca}^{2+}$ levels (Fura-2/AM; Life Technologies). Neurons were differentiated from NPCs and functionally characterized by means of whole-cell voltage- and current-clamp recordings. Complete and detailed description of methods and materials can be found in the SUPPLEMENTAL METHODS AND MATERIALS section. 


\section{RESULTS}

Based on our findings of altered bioenergetic properties and mitochondrial function in primary dermal fibroblasts of depressed patients (1), we were interested in the identification and characterization of MDD-associated pathomechanisms in neural cells. Thus, we used primary skin fibroblasts of a cohort of patients diagnosed with major depressive disorder as well as fibroblasts from non-depressed controls of same age and gender as used in our earlier study (1), and reprogrammed them to induced pluripotent stem cells (iPSCs) by transient episomal transduction according to the Yamanaka protocol (Figure 1A) (14, 15). Pluripotency of iPSCs was validated by PluriTest analysis, a bioinformatics assay for the quality assessment of iPSCs by transcription profiling based on next-generation sequencing data (18) obtained from the respective iPSC clones. The PluriTest analysis revealed that 13 of the iPSC clones used in this study could be considered as pluripotent. Three others were very close to the empirical threshold (pluripotency >1424; novelty <2.5) (Figure 1B and Table 1). The iPSC colonies were differentiated to early neural progenitor cells (NPCs) within 7 days after culture medium was changed from mTeSR to neural induction medium. Further maturation of NPCs was allowed for 5 passages before cells were stained for neural progenitor markers SOX2 and PAX6 (Figure 2). Table 2 presents the percentage of cells positively labelled with the respective antibody (Table 2) and indicates that the majority of the cells co-expresses both NPC markers and can be regarded as neural progenitors. The three cell lines which failed the PluriTest analysis likewise showed prominent expression of the neuronal progenitor markers Pax6 and SOX2, thus we decided to not exclude these cell lines from our study. To seek for functional differences between NPCs from depressed and nondepressed subjects, we analyzed bioenergetic properties and mitochondrial function.

\section{Mitochondrial membrane potential}

The mitochondrial membrane potential (MMP) is generated by the guided flux of electrons along the electron transport chain (ETC) along with the translocation of protons over the inner mitochondrial membrane. The resulting proton gradient is the primary force, which drives the synthesis of ATP at complex $\mathrm{V}$ within the mitochondrial oxidative phosphorylation system (OXPHOS). Acting as a biophysical integrator, the MMP could serve as an indicator for the bioenergetic state, uncovering a potential bioenergetic misbalance, which may result from mitochondrial dysfunction. The cationic and 
lipophilic fluorescent dye JC-1 accumulates in the mitochondrial membrane to an extent, which is dependent on the strength of the electric field. In negatively charged (i.e. highly energized) mitochondria, JC-1 molecules accumulate and form red fluorescing aggregates, while the fluorescence changes to green, when the dye molecules disaggregate into monomers in response to dissipation of the transmembrane potential. The ratio of the fluorescence signals emitted by the two states of JC-1 is then analyzed as a measure of the MMP. We found that NPCs from depressed patients presented a significantly lower red/green ratio when compared to the control NPCs, pointing to a more depolarized MMP in patient cells (MDD $1.70 \pm 0.065$ vs. Cntrl $1.84 \pm 0.092, \mathrm{p}=0.0268$, paired t-test) (Figure 3). Since stressful life events are associated with the onset and severity of major depression, we additionally set out to investigate whether aberrant adaptive responses to stressors would be detectable at the cellular/mitochondrial level. Thus, we grew the NPCs under control conditions, but also treated them with the synthetic glucocorticoid dexamethasone (DEX, $1 \mu \mathrm{M}, 1$ week) to apply hormonal stress. DEX-treatment significantly increased the MMP in NPCs from depressed patients (MDD $1.96 \pm$ 0.079 ) but did not reach the level of significance in control NPCs (Cntrl $2.04 \pm 0.128)$. The difference in MMP between untreated MDD and control NPCs vanished after hormonal stress (Figure 3).

\section{Mitochondrial oxidative phosphorylation system (OXPHOS) - Respirometry}

The mitochondrial oxidative system (OXPHOS) generates ATP by utilizing the proton gradient, which is established by sequential redox reactions along the electron transport chain (ETC). The consumption of molecular oxygen by accepting the electrons delivered by the ETC at complex IV can be used as a readout for the function and performance of the OXPHOS. We used the Seahorse Flux Analyzer technology for direct measurement of the oxygen consumption rate (OCR) in dependence on specific compounds which affect the activity of the individual molecular respiratory complexes. Figure 4A depicts a representative Seahorse measurement using the Mito Stress Kit. We assessed the function of the ETC and OXPHOS in mitochondria of both MDD and control NPCs and analysed the basal mitochondrial respiration, the oxygen consumption related to ATP-synthesis, and the maximal respiration. Moreover, the proton-leak and the fraction of oxygen consumed by non-mitochondrial processes in MDD and control NPCs were also recorded. We found a significantly reduced basal respiration in MDD NPCs (MDD 88.49 \pm 3.42 . vs. Cntrl $103.72 \pm 4.52 ; \mathrm{p}=0.0089$, paired t-test), as 
well as a reduced proton leak (MDD $16.63 \pm 0.79$ vs. Cntrl 19.56 $\pm 1.01 ; \mathrm{p}=0.0078$, paired t-test) and non-mitochondrial oxygen consumption (MDD $25.54 \pm 1.20$ vs. Cntrl $30.82 \pm 1.81 ; p=0.0195$, Wilcoxon matched-paired signed rank test) (Figure 4B). Other respiratory parameters were not significantly different between MDD and control NPCs (Figure 4C). To investigate the impact of hormonal stress on mitochondrial OXPHOS, we treated MDD and control NPCs with DEX $(1 \mu \mathrm{M}, 1$ week), but did not find significant differences between the groups in any of their respiratory parameters (Figure 4B and C).

\section{Cellular ATP-content and cytosolic $\mathrm{Ca}^{2+}$ level}

Although we found a reduced basal respiration, as well as reduced proton leak and non-mitochondrial respiration in MDD NPCs compared with the controls, the cellular ATP content was not different between the two groups (Figure 5A). Moreover, DEX-treatment had no impact on ATP-content, neither within nor between groups. As an additional measure for metabolic/bioenergetic activity and mitochondrial function, we compared the cytosolic $\mathrm{Ca}^{2+}$ levels in MDD and control NPCs by Fura-2 $\mathrm{Ca}^{2+}$ imaging. However, we did not detect a significant difference in cytosolic $\mathrm{Ca}^{2+}$ between MDD or control NPCs, neither under control nor hormonal stress conditions ( $1 \mu \mathrm{M}$ DEX, 1 week) (Figure 5B).

\section{Functional characteristics of iPS-neurons}

After having identified differences in mitochondrial parameters and bioenergetic function between NPCs derived from depressed patients or non-depressed controls, we were interested whether differential functional phenotypes can be associated also with iPS-neurons from MDD or control subjects. To this end, we differentiated NPC lines from eight MDD patients and matching controls to iPS-neurons. iPS-neurons showed clear bipolar or multipolar neuronal morphology (Figure 6A) and expressed the typical neuronal markers class III beta-tubulin, MAP2 and NEUN (Figure 6B). Moreover, they were immunopositive for the specific synaptic proteins VGLUT1 and SYP after day 21 in vitro (DIV 21) (Figure 6C and D). To functionally characterize and compare electrophysiological properties of iPS-neurons, we performed whole-cell voltage-clamp and current-clamp recordings of iPS-neurons during their $4^{\text {th }}$ week of differentiation and analyzed their passive biophysical as well as their active neuronal properties. Figure 7 shows exemplary whole-cell recordings of a typical iPS neuron. In total, we recorded 333 neurons derived and differentiated from the eight MDD patients and 
eight non-depressed controls. We found that the membrane capacitance of iPS-neurons from MDD patients, which was compensated as C-slow by the inbuilt capacitance compensation algorithm of the EPC 10 amplifier, was significantly smaller compared with non-depressed control neurons ([pF] MDD $15.75 \pm 0.76$ vs. Cntrl $18.12 \pm 0.9 ; p=0.044, t$-test, unpaired) (Figure 8A). Since C-slow is associated with the amount of membrane material of the recorded cell, the capacitance parameter can be regarded as a measure for cell size. Thus, this observation indicates that iPS-neurons from MDD patients are electrically smaller than from non-depressed controls. Depending on the morphology and architecture of the cell, this parameter is likely to be associated with the size of the soma and proximal neurite compartments only. The series resistance was not different between the two groups ([M $\Omega]$ MDD $23.09 \pm 1.035$ vs. Cntrl $24.79 \pm 1.11 ; p=0.26, t$-test, unpaired). Interestingly, we found that the resting membrane potential measured directly after establishing the whole-cell configuration was different between the groups: MDD iPS-neurons had a significantly lower (i.e. more depolarized) resting membrane potential than iPS-neurons derived from the control subjects ([mV] MDD $-23.68 \pm 0.84$ vs. Cntrl -26.68 $\pm 1.02 ; p=0.024, t$-test, unpaired, with Welch's correction) (Figure 8B). In current-clamp mode, the neurons were able to fire action potentials (APs) when the membrane potential was adjusted to approximately $-80 \mathrm{mV}$ by current injection and then depolarized by modulating the direct current. The mean current to adjust the membrane potential to $-80 \mathrm{mV}$ was not different between MDD and non-depressed control iPS-neurons ([pA] MDD $-84.08 \pm 5.214$ vs. Cntrl $-81.14 \pm 5.29 ; p=0.69, t$ test, unpaired) (Figure 9A). iPS-neurons generated APs when the depolarizing current drove the membrane potential above a threshold of $-28.79 \pm 0.95 \mathrm{mV}$ in MDD or $-28.97 \pm 0.93 \mathrm{mV}$ in nondepressed control cells, indicating that this threshold also was not different between groups. In voltage-clamp experiments, voltage-activated sodium inward currents and potassium outward currents were induced by stepwise depolarization of the membrane potential starting at a $\mathrm{V}_{\text {hold }}$ of $-80 \mathrm{mV}$. Analysis of current/voltage (I/V) relationships revealed that the maximal amplitudes of sodium or potassium currents were not different between MDD and non-depressed iPS-neurons. In addition, we characterized spontaneous electrical activity of cultured iPS-neurons by current-clamp recording and adjusting the resting membrane potential to about $-50 \mathrm{mV}$ by direct current injection. Under this condition, we found that a significantly higher proportion of MDD (21.74\%) derived iPS-neurons 
were spontaneously active and generated APs when compared with cells reprogrammed from nondepressed controls $(11.11 \%)$ indicating an increased activity of MDD neurons ( $\mathrm{p}=0.016$, Fisher's exact test) (Figure 9B).

\section{DISCUSSION}

Major depression, or more general depressive syndrome, is a severe psychiatric disease which manifests in emotional, somatic and cognitive symptoms. The underlying pathomechanisms are manifold and complex and not completely understood. Moreover, the fact that therapeutic approaches using common antidepressants often show insufficient efficacy and low response rates, strengthens the need for deeper investigation of involved mechanisms. In order to identify and characterize molecular pathomechanisms of depression, we established a human cellular model, which is based on dermal fibroblasts derived from skin biopsies of depressed patients and non-depressed controls (1). In detail, we used induced pluripotent stem cell-derived neural progenitor cells reprogrammed from the skin fibroblasts to investigate cellular and molecular function of depressed cells and focused on possible bioenergetic differences in NPC which might be associated with depressive syndrome.

The quality of the reprogrammed stem cells was analyzed by PluriTest (18), a bioinformatics-based approach which analyzes the genome-wide transcription profile and provides a classification of the reprogrammed cells with regard to pluripotency and novelty. 13 of the tested iPS cell lines used in this study were above threshold and were regarded as being pluripotent. Three others failed this criterion but were very close to the empirical threshold. We decided to not exclude these cell lines from our study, since the NPCs developed from these iPSC lines showed prominent expression of the neuronal progenitor markers Pax6 and SOX2 and were not different in this regard from the other NPC clones (19).

Analysis of the mitochondrial function of reprogrammed human NPCs revealed a reduced basal respiration and a more depolarized mitochondrial membrane potential in MDD NPCs. This functional phenotype indicates a significant mitochondrial dysfunction which was already evident in the original cells (i.e. fibroblasts) of depressed patients (1). Other functional parameters of the bioenergetic status of the NPCs such as the maximal respiration, cellular ATP content or cytosolic $\mathrm{Ca}^{2+}$ level were not found to be significantly different between the MDD and the control group, although the cytosolic 
$\mathrm{Ca}^{2+}$ level showed a trend of being higher in NPCs of depressed patients $(\mathrm{p}=0.07)$. The altered functional phenotype might in part be explained by an altered metabolic dependence of NPCs. Whereas fibroblasts rely highly on OXPHOS for ATP production, NPCs gain a significant part of their energy via the glycolytic pathway $(20,21)$. Thus, potential deficits in their mitochondrial OXPHOS and ETC activity can be compensated by the predominantly generation of energy through the glycolytic metabolism of glucose. Consistent with this, the cellular ATP content was not significantly different between MDD and control NPCs. The dependence of NPCs on the glycolytic pathway was also demonstrated by their inability to use galactose as a substrate and grow under glucose-free conditions. In general, without glucose, cells are unable to generate ATP by glycolysis, but are forced to meet their energetic demands by mitochondrial OXPHOS (in the presence of galactose and glutamine) (22-24). Since NPCs rely mostly on their glycolytic activity for ATP production $(20,21)$, they cannot survive under such a metabolic stress. Thus, when testing for cellular resilience of MDD and control NPCs, we challenged the cells with dexamethasone to apply hormonal stress on mitochondrial function only, but refrained from metabolic stress, which we additionally used for characterization of fibroblasts (1). Interestingly, differences in mitochondrial function between MDD and control groups vanished upon the treatment of NPCs with dexamethasone, demonstrating that mitochondrial respiration and function in depressed and non-depressed cells reached similar levels under hormonal stress. This was also observed in fibroblasts of depressed patients in our earlier study (1).

The MDD patients were asked to participate in our study and to donate a skin biopsy at the end of their inpatient stay at the Department of Psychiatry and Psychotherapy of the University of Regensburg (1). At this time, the patients received antidepressant medication and were nearly in remission. Importantly, after several cell divisions of fibroblasts in vitro, the confounding variability in these samples on the basis of the subjects' hormones, life style or medication use, should be virtually eliminated (9). Thus, the differences in mitochondrial function we have found in our study might be associated rather with a trait than with the state of depression.

The etiology of depression is associated with various environmental risk factors such as stress, childhood maltreatment experiences, and stressful life events which directly influence physiological 
processes and mental health of an individual, immediately and in later life. These threats are suggested to affect gene-environment $(\mathrm{GxE})$ interactions and epigenetics by altering chromatin (both in terms of global/genomewide changes as well as promoter-specific changes) and lead to altered molecular patterns observed in depression (25-27). In this context, it is important to consider the question of how much of the epigenetic memory is erased during the reprogramming process, since a total loss of epigenetic markers would pose a challenge to the approach of studying neuropsychiatric disorders by using induced pluripotent stem cells as a model system. Others reported that iPS cells retain a transcriptional memory of the original cells upon reprogramming (28-30). In this line of evidence, we observed mitochondrial dysfunction in fibroblasts of depressed patients (1) but also in iPSC-derived NPCs reprogrammed from exactly these fibroblasts. Our data are in favor of the hypothesis that epigenetic changes present in the somatic cells of the patient, such as in skin fibroblasts, are relevant in the context of modeling a neuropsychiatric disease. Besides epigenetic factors, the etiology of depression is also dependent on the genetic architecture of an individual, including certain risk factors in the genome $(31,32)$. However, analysis of such risk variants in the genome of our patient's cells is an important next step in our search for molecular pathomechanisms of depression.

So far, depression is regarded as illness of the mind as well as of the body. Following this hypothesis, we have detected mitochondrial dysfunction in fibroblasts and in reprogrammed NPCs of depressed patients. In order to search for neuron-specific disease-related phenotypes and to deepen our understanding of the pathomechanisms involved in depression, we differentiated the NPCs of depressed and non-depressed individuals to iPS-neurons, and performed electrophysiological characterization of the biophysical properties of the iPS-neurons by means of whole-cell patch-clamp recording. We recorded from the iPS-neurons after 21 days of differentiation and found that the resting membrane potential was significantly more positive in iPS-neurons derived from depressed patients compared to non-depressed controls. Moreover, MDD neurons showed a smaller membrane capacitance which is indicative for smaller soma size or altered geometry of the neuron (33-35). Intriguingly, iPS-neurons from depressed patients showed a higher spontaneous activity within the neuronal network in vitro. In general, a lower resting membrane potential as well as a smaller cell size which is indicated by lower membrane capacitance, could be a result of reduced energy availability in 
consequence of mitochondrial dysfunction (36), or due to a delayed neural development, since a hyperpolarized resting membrane potential as well as cell size is associated with neuronal maturation $(37,38)$. The increased network activity analyzed as spontaneous activity of a neuron when recorded in current-clamp mode is an intriguing observation which needs deeper investigation. Altered neurite growth and morphology of iPS-neurons derived from depressed patients which were resistant to serotonin reuptake inhibitors have recently been demonstrated and were associated with a reduced expression of the Protocadherin alpha genes in these patients (39). How this altered activity in iPSneurons in vitro relates to interregional network activity and functional connectivity dynamics in vivo $(40,41)$, is not known, but an interesting and important objective for future investigations.

Moreover, neurons made from psychiatric patients can be used to screen for new therapeutics and elucidate the unknown mechanisms through which current drugs may exert their action. This approach may lead to the discovery of new drug targets and new insights into the molecular biology and pathophysiology of depression. 


\section{ACKNOWLEDGEMENT}

The authors would like to thank Richard Warth for providing access to the Seahorse device. The excellent technical assistance by Tatjana Jahner is gratefully acknowledged.

\section{FUNDING}

The work has been supported by the Deutsche Forschungsgemeinschaft (DFG, German Research Foundation) project number 422182557 to C.H.W., and GRK2174 to C.H.W., K.K. and I.C.), the Bavarian State Ministry of Science and the Arts (Bavarian Research Networks ForIPS and ForInter, grants to M.J.R.), and the BMBF (Research Grant No. 01EE1401B to T.C.B. and R.R.). 


\section{REFERENCES}

1. Kuffner K, Triebelhorn J, Meindl K, Benner C, Manook A, Sudria-Lopez D, et al. (2020): Major Depressive Disorder is Associated with Impaired Mitochondrial Function in Skin Fibroblasts. Cells. 9.

2. Klengel T, Binder EB (2013): Gene-environment interactions in major depressive disorder. Can J Psychiatry. 58:76-83.

3. Hasler G (2010): Pathophysiology of depression: do we have any solid evidence of interest to clinicians? World Psychiatry. 9:155-161.

4. Manji H, Kato T, Di Prospero NA, Ness S, Beal MF, Krams M, et al. (2012): Impaired mitochondrial function in psychiatric disorders. Nat Rev Neurosci. 13:293-307.

5. Gardner A, Boles RG (2011): Beyond the serotonin hypothesis: mitochondria, inflammation and neurodegeneration in major depression and affective spectrum disorders. Prog Neuropsychopharmacol Biol Psychiatry. 35:730-743.

6. Klinedinst NJ, Regenold WT (2015): A mitochondrial bioenergetic basis of depression. $J$ Bioenerg Biomembr. 47:155-171.

7. Picard M, McEwen BS (2014): Mitochondria impact brain function and cognition. Proc Natl Acad Sci U S A. 111:7-8.

8. Verhoeven JE, Revesz D, Wolkowitz OM, Penninx BW (2014): Cellular aging in depression: Permanent imprint or reversible process?: An overview of the current evidence, mechanistic pathways, and targets for interventions. Bioessays. 36:968-978.

9. Garbett KA, Vereczkei A, Kalman S, Wang L, Korade Z, Shelton RC, et al. (2015):

Fibroblasts from patients with major depressive disorder show distinct transcriptional response to metabolic stressors. Transl Psychiatry. 5:e523.

10. Gardner A, Johansson A, Wibom R, Nennesmo I, von Dobeln U, Hagenfeldt L, et al. (2003): Alterations of mitochondrial function and correlations with personality traits in selected major depressive disorder patients. J Affect Disord. 76:55-68. 
11. Karabatsiakis A, Bock C, Salinas-Manrique J, Kolassa S, Calzia E, Dietrich DE, et al. (2014): Mitochondrial respiration in peripheral blood mononuclear cells correlates with depressive subsymptoms and severity of major depression. Transl Psychiatry. 4:e397.

12. Hroudova J, Fisar Z, Kitzlerova E, Zverova M, Raboch J (2013): Mitochondrial respiration in blood platelets of depressive patients. Mitochondrion. 13:795-800.

13. Sjovall F, Ehinger JK, Marelsson SE, Morota S, Frostner EA, Uchino H, et al. (2013): Mitochondrial respiration in human viable platelets--methodology and influence of gender, age and storage. Mitochondrion. 13:7-14.

14. Takahashi K, Tanabe K, Ohnuki M, Narita M, Ichisaka T, Tomoda K, et al. (2007): Induction of pluripotent stem cells from adult human fibroblasts by defined factors. Cell. 131:861-872.

15. Okita K, Matsumura Y, Sato Y, Okada A, Morizane A, Okamoto S, et al. (2011): A more efficient method to generate integration-free human iPS cells. Nat Methods. 8:409-412.

16. Inoue H, Nagata N, Kurokawa H, Yamanaka S (2014): iPS cells: a game changer for future medicine. EMBO J. 33:409-417.

17. Takahashi K, Yamanaka S (2013): Induced pluripotent stem cells in medicine and biology. Development. 140:2457-2461.

18. Schulze M, Hoja S, Winner B, Winkler J, Edenhofer F, Riemenschneider MJ (2016): Model Testing of PluriTest with Next-Generation Sequencing Data. Stem Cells Dev. 25:569-571.

19. Zhang XQ, Huang CT, Chen J, Pankratz MT, Xi JJ, Li J, et al. (2010): Pax6 Is a Human Neuroectoderm Cell Fate Determinant. Cell Stem Cell. 7:90-100.

20. Lorenz C, Lesimple P, Bukowiecki R, Zink A, Inak G, Mlody B, et al. (2017): Human iPSCDerived Neural Progenitors Are an Effective Drug Discovery Model for Neurological mtDNA Disorders. Cell Stem Cell.

21. Zheng X, Boyer L, Jin M, Mertens J, Kim Y, Ma L, et al. (2016): Metabolic reprogramming during neuronal differentiation from aerobic glycolysis to neuronal oxidative phosphorylation. Elife. 5 . 
22. Marroquin LD, Hynes J, Dykens JA, Jamieson JD, Will Y (2007): Circumventing the Crabtree effect: replacing media glucose with galactose increases susceptibility of HepG2 cells to mitochondrial toxicants. Toxicol Sci. 97:539-547.

23. Robinson BH, Petrova-Benedict R, Buncic JR, Wallace DC (1992): Nonviability of cells with oxidative defects in galactose medium: a screening test for affected patient fibroblasts. Biochem Med Metab Biol. 48:122-126.

24. Bustamante E, Pedersen PL (1977): High aerobic glycolysis of rat hepatoma cells in culture: role of mitochondrial hexokinase. Proc Natl Acad Sci U S A. 74:3735-3739.

25. Sun H, Kennedy PJ, Nestler EJ (2013): Epigenetics of the depressed brain: role of histone acetylation and methylation. Neuropsychopharmacology. 38:124-137.

26. Nestler EJ (2014): Epigenetic mechanisms of depression. JAMA Psychiatry. 71:454-456.

27. Uchida S, Yamagata H, Seki T, Watanabe Y (2018): Epigenetic mechanisms of major depression: Targeting neuronal plasticity. Psychiatry Clin Neurosci. 72:212-227.

28. Doi A, Park IH, Wen B, Murakami P, Aryee MJ, Irizarry R, et al. (2009): Differential methylation of tissue- and cancer-specific $\mathrm{CpG}$ island shores distinguishes human induced pluripotent stem cells, embryonic stem cells and fibroblasts. Nat Genet. 41:1350-1353.

29. Lister R, Pelizzola M, Kida YS, Hawkins RD, Nery JR, Hon G, et al. (2011): Hotspots of aberrant epigenomic reprogramming in human induced pluripotent stem cells. Nature. 471:68-73.

30. Ohi Y, Qin H, Hong C, Blouin L, Polo JM, Guo T, et al. (2011): Incomplete DNA methylation underlies a transcriptional memory of somatic cells in human iPS cells. Nat Cell Biol. 13:541-549.

31. Wray NR, Ripke S, Mattheisen M, Trzaskowski M, Byrne EM, Abdellaoui A, et al. (2018):

Genome-wide association analyses identify 44 risk variants and refine the genetic architecture of major depression. Nat Genet.

32. Gratten J, Wray NR, Keller MC, Visscher PM (2014): Large-scale genomics unveils the genetic architecture of psychiatric disorders. Nat Neurosci. 17:782-790.

33. Lindau M, Neher E (1988): Patch-clamp techniques for time-resolved capacitance measurements in single cells. Pflugers Arch. 411:137-146. 
34. Hernández-Balaguera E, Vara H, Polo JL (2018): Identification of Capacitance Distribution in Neuronal Membranes from a Fractional-Order Electrical Circuit and Whole-Cell Patch-Clamped Cells. Journal of The Electrochemical Society. 165:G3104-G3111.

35. Kim MH, von Gersdorff H (2010): Extending the realm of membrane capacitance measurements to nerve terminals with complex morphologies. J Physiol. 588:2011-2012.

36. Vaarmann A, Mandel M, Zeb A, Wareski P, Liiv J, Kuum M, et al. (2016): Mitochondrial biogenesis is required for axonal growth. Development. 143:1981-1992.

37. Pre D, Nestor MW, Sproul AA, Jacob S, Koppensteiner P, Chinchalongporn V, et al. (2014): A time course analysis of the electrophysiological properties of neurons differentiated from human induced pluripotent stem cells (iPSCs). PLoS One. 9:e103418.

38. Fang D, Qing Y, Yan S, Chen D, Yan SS (2016): Development and Dynamic Regulation of Mitochondrial Network in Human Midbrain Dopaminergic Neurons Differentiated from iPSCs. Stem Cell Reports. 7:678-692.

39. Vadodaria KC, Ji Y, Skime M, Paquola AC, Nelson T, Hall-Flavin D, et al. (2019): Altered serotonergic circuitry in SSRI-resistant major depressive disorder patient-derived neurons. Molecular Psychiatry.

40. Helm K, Viol K, Weiger TM, Tass PA, Grefkes C, Del Monte D, et al. (2018): Neuronal connectivity in major depressive disorder: a systematic review. Neuropsychiatr Dis Treat. 14:27152737.

41. Drysdale AT, Grosenick L, Downar J, Dunlop K, Mansouri F, Meng Y, et al. (2017): Restingstate connectivity biomarkers define neurophysiological subtypes of depression. Nat Med. 23:28-38. 


\section{FIGURE LEGENDS}

Figure 1. Generation of iPSC-based model of MDD. (A) Patient-derived dermal fibroblasts were reprogramed into iPSCs and subsequently differentiated into neurons. Representative images of fibroblasts, iPSCs, NPCs and iPS-neurons expressing corresponding cell specific markers. Scale bar indicates $20 \mu \mathrm{m}$. (B) Characterization of 16 pluripotent cell lines (black dots) from this study using PluriTest. Kernel density estimations for iPSCs (yellow to red), and non-pluripotent stem cells (shades of blue/grey) are shown. Black dashed lines indicate the empirical thresholds for novelty and pluripotency. High quality cells should be located in the upper left quadrant, with a pluripotency score above the threshold and a novelty score below the threshold. It can be seen that the empirical thresholds detect high quality pluripotent cells from this study, but that this population still contains some cells with reduced pluripotency.

Figure 2. Representative images of NPCs differentiated from control and MDD patients show that majority of the cells are expressing typical neural progenitor cell markers PAX6 and SOX2. Scale bar indicates $20 \mu \mathrm{m}$.

Figure 3. Mitochondrial membrane potential of NPCs. Red/green (JC-1 aggregate/monomer) ratios of control and MDD NPCs under non-treated and DEX-treated ( $1 \mu \mathrm{M}, 7$ days), conditions. Significant differences were found for non-treated control vs MDD cells. \# and $\S$ indicate a significant effect of treatment. Bar graphs show mean red/green ratios \pm SEM; control $n=8$, MDD $n=8$.

Figure 4. Oxygen consumption rates (OCR) of control (indicated in blue) and MDD (indicated in orange) NPCs under non-treated conditions, as well as after one week of DEX $(1 \mu \mathrm{M})$ stress. (A) Representative OCR measurement during the Mito Stress Test for MDD and control NPCs. (B) Basal respiration, proton leak, and non-mitochondrial respiration were significantly reduced in MDD derived cells. Bar graphs show normalized mean OCR values \pm SEM; Control $n=8$, MDD $n=8$. Significant differences between MDD and non-depressive controls are indicated with *. 
Figure 5. ATP and basal $\mathrm{Ca}^{2+}$ calcium levels were not significantly altered in MDD patient NPCs. (A) ATP content in control and MDD NPCs under non-treated and DEX-treated (1 $\mu \mathrm{M}, 7$ days) conditions. Bar graphs show normalized mean RLU values \pm SEM. (B) Shown are the Fura-2 340 $\mathrm{nm} / 380 \mathrm{~nm}$ fluorescence ratios of non-depressed control and MDD NPC lines under non-treated and DEX-treated conditions. Bar graphs show mean ratios $(340 \mathrm{~nm} / 380 \mathrm{~nm}$; ratios $\pm \mathrm{SEM})$. Control $\mathrm{n}=8$, MDD $n=8$.

Figure 6. Characterization of iPS-neurons after 3-4 weeks in the culture. (A) Neurons differentiated from NPCs show clear bipolar or -multipolar neuronal morphology. (B) Representative images of iPSneurons expressing typical neuronal markers such as class III beta-tubulin (TUJ1), microtubule associated protein 2 (MAP2), and neuronal nuclear protein (NEUN). Scale bar indicates $20 \mu \mathrm{m} .($ C, D) Formation of glutamatergic synapses in iPS-neurons after 3-4 weeks in the culture. VGLUT1 and synaptophysin (SYP) immunostaining visualizes synaptic vesicle clusters (green; white arrows). Scale bar indicates $10 \mu \mathrm{m}$.

Figure 7. Electrophysiological characterization of iPSC-derived neurons. (A) Exemplary differential interference contrast photomicrograph (400x) of a cultured iPSC-derived neuron (DIV 21) from a MDD patient. A patch-pipette is attached to the soma of the cell. (B) Whole-cell recording in the current-clamp mode allows recording of the membrane potential. Injection of a supra-threshold depolarizing current induced action potentials. (C) Whole-cell recording in the voltage-clamp mode showed voltage-activated transient $\mathrm{Na}^{+}$-inward $\left(\mathrm{I}_{\mathrm{Na}+}\right)$ and delayed $\mathrm{K}^{+}$-outward currents $\left(\mathrm{I}_{\mathrm{K}+}\right)$ following a pulse protocol stepping from $\mathrm{V}_{\text {hold }}=-80 \mathrm{mV}$ to more depolarized potentials $(-70$ to $+40 \mathrm{mV})$. An exemplary current/voltage relationship for voltage-activated $\mathrm{Na}^{+}\left(\mathrm{I}_{\mathrm{Na}+}\right.$; dark grey filled circles) and $\mathrm{K}^{+}$ currents $\left(\mathrm{I}_{\mathrm{K}+}\right.$; light grey filled circles) is presented in (D).

Figure 8. Passive electrophysiological properties of iPSC-derived neurons from MDD patients and non-depressed controls. (A) Analysis of the membrane capacitance as a measure of the size of the recorded cell revealed a significantly smaller mean value in cells derived from MDD patients. (B) The 
resting membrane potential measured in the current-clamp mode was less hyperpolarized in MDD iPS-derived neurons. Values are presented as mean \pm SEM.

Figure 9. Active electrophysiological properties of iPSC-derived neurons. (A) Amplitudes of transient $\mathrm{Na}^{+}$inward currents $\left(\mathrm{I}_{\mathrm{Na}+} ;\right.$ maximum current $)$ and delayed $\mathrm{K}^{+}$outward currents $\left(\mathrm{I}_{\mathrm{K}+} ;\right.$ at a voltage jump to $+20 \mathrm{mV}$ ) were not different between MDD and healthy control neurons. Values are presented as mean \pm SEM. (B) The fraction of spontaneously active iPS-neurons, which fire action potentials at the adjusted membrane potential of $-50 \mathrm{mV}$, was significantly higher in the MDD patient group. 


\begin{tabular}{cccc}
\hline Sample & Result & Pluripotency $(>1424)$ & Novelty $(<2.5)$ \\
\hline CON1 & Pluripotent & 1540.3 & 1.99 \\
MDD1 & Not Pluripotent & 1336.6 & 2.32 \\
CON2 & Not Pluripotent & 1403.1 & 2.44 \\
MDD2 & Pluripotent & 1513.4 & 2.45 \\
CON3 & Pluripotent & 1426.4 & 2.49 \\
MDD3 & Pluripotent & 1473.5 & 2.34 \\
CON4 & Not Pluripotent & 1392.2 & 2.41 \\
MDD4 & Pluripotent & 1598.5 & 2.25 \\
CON5 & Pluripotent & 1442.1 & 2.24 \\
MDD5 & Pluripotent & 1524.2 & 2.20 \\
CON6 & Pluripotent & 1457.6 & 2.27 \\
MDD6 & Pluripotent & 1488.8 & 2.31 \\
CON7 & Pluripotent & 1597.9 & 2.33 \\
MDD7 & Pluripotent & 1504.7 & 2.38 \\
CON8 & Pluripotent & 1521.2 & 2.49 \\
MDD8 & Pluripotent & 1661.4 & 2.32 \\
\hline
\end{tabular}

Table 1. PluriTest results from all cells used in this study. 


\begin{tabular}{ccc}
\hline Cell line & PAX6 positive (\%) & SOX2 positive (\%) \\
\hline CON1 & 87.6 & 86.0 \\
MDD1 & 90.8 & 96.3 \\
CON2 & 90.7 & 90.0 \\
MDD2 & 82.2 & 85.4 \\
CON3 & 84.6 & 85.6 \\
MDD3 & 91.7 & 91.4 \\
CON4 & 86.4 & 90.8 \\
MDD4 & 84.3 & 86.9 \\
CON5 & 90.4 & 93.0 \\
MDD5 & 87.8 & 89.6 \\
CON6 & 93.1 & 92.9 \\
MDD6 & 91.8 & 89.2 \\
CON7 & 91.7 & 91.7 \\
MDD7 & 89.1 & 90.4 \\
CON8 & 88.6 & 89.7 \\
MDD8 & 88.3 & 90.2 \\
\hline
\end{tabular}

Table 2. Generation of PAX6/SOX2 positive NPCs from human iPSCs via 


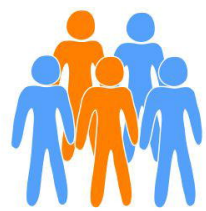

Neural progenitor cells

Neural differentiation

2 wks

Patient and control-derived iPSCs

Fibroblasts
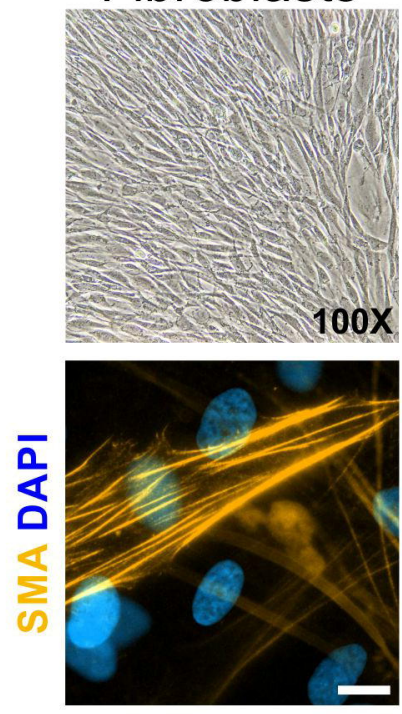

iPSCs
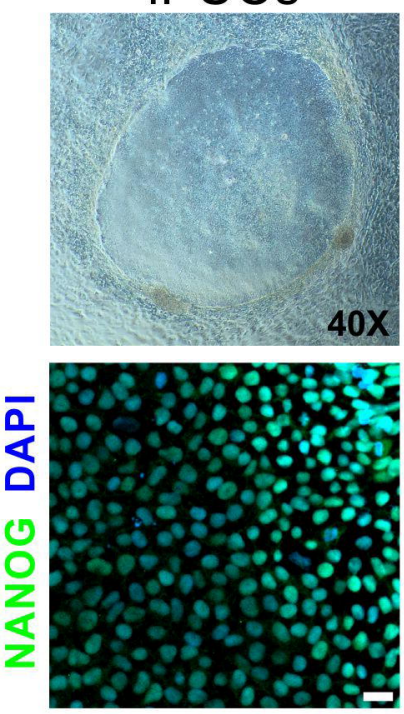

4 wks

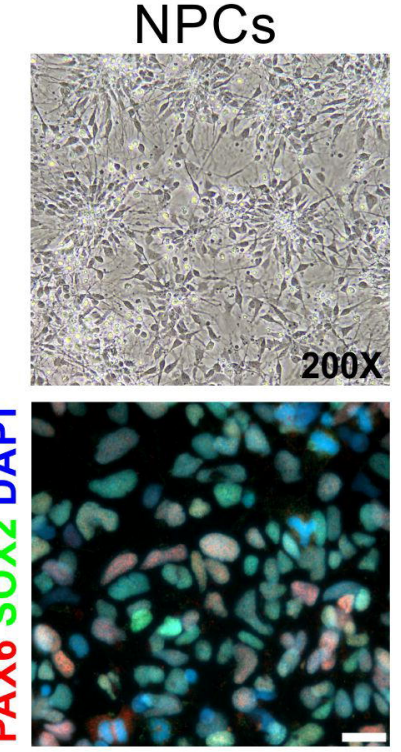

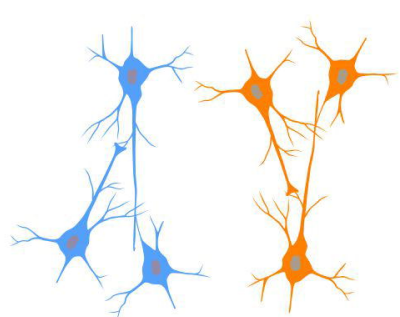

iPS-neurons
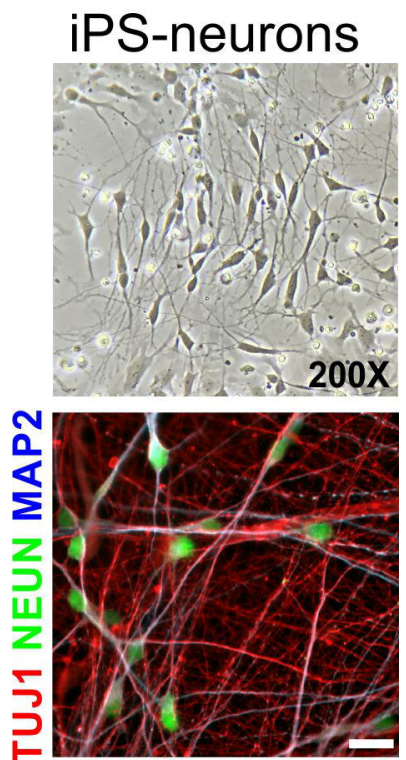

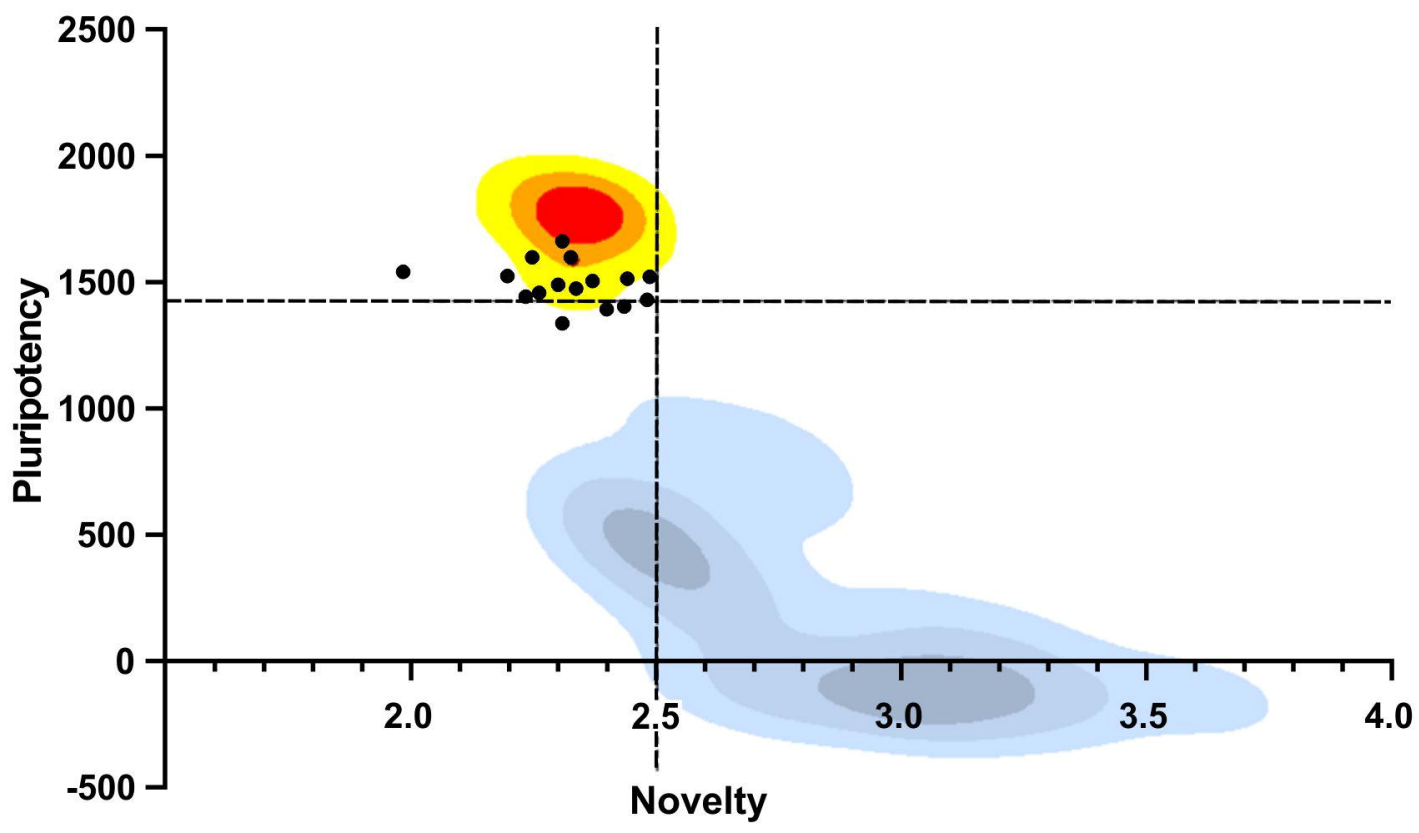

Fig 1 


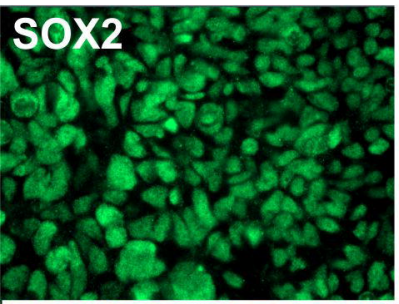

\section{DAPI} xic e-3

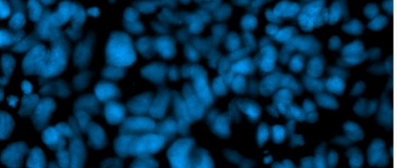
rocron
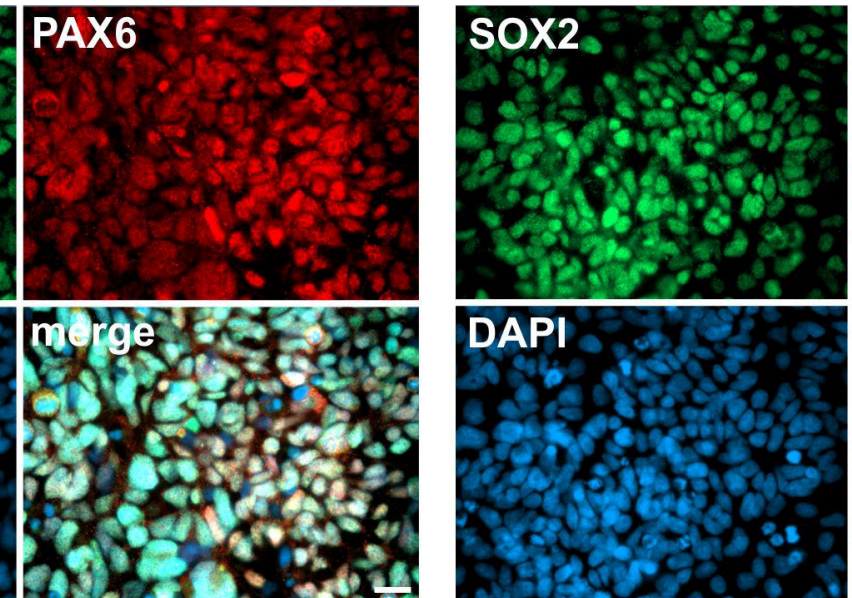

DAPI

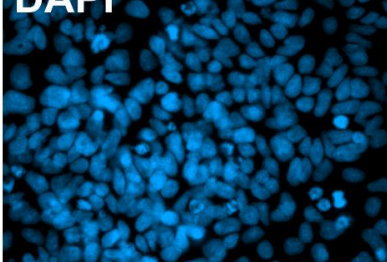

\section{PAX6}

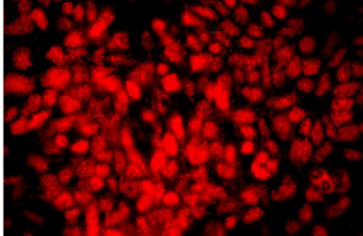
merges $3,2,3$ - 1 S 10

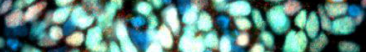
. Q

Fig 2. 
Mitochondrial Membrane Potential
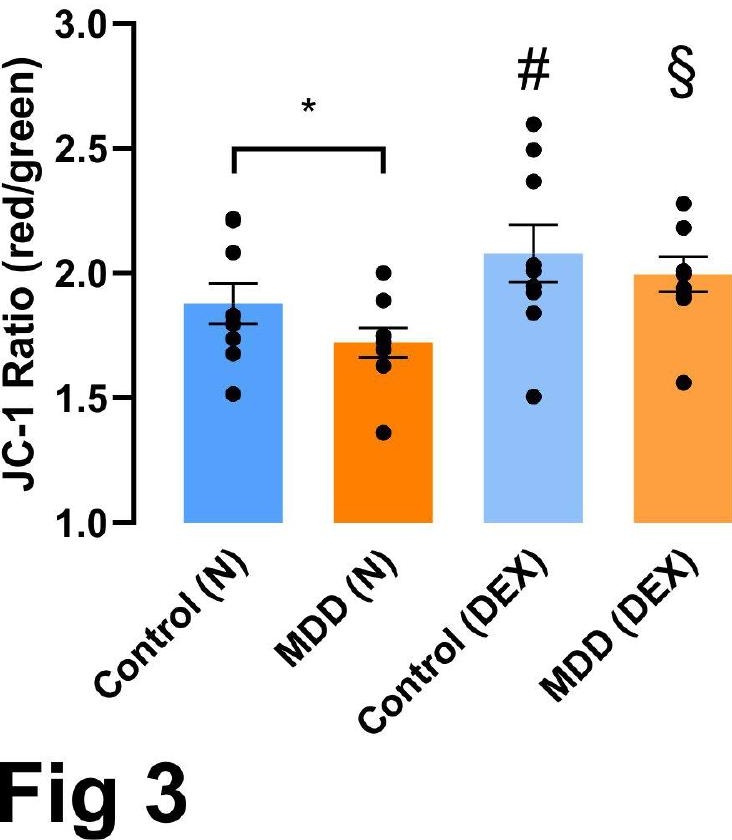
A

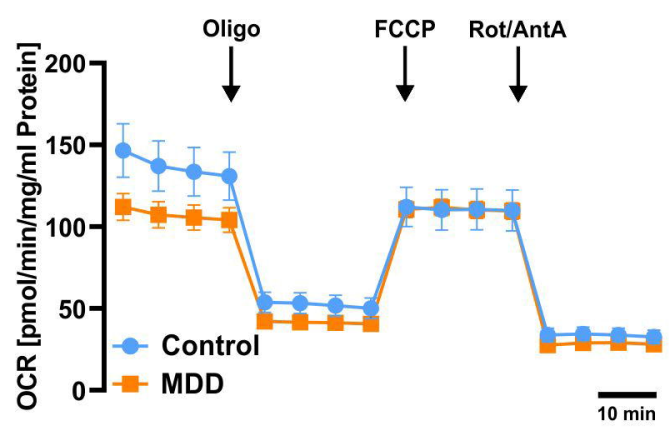

B
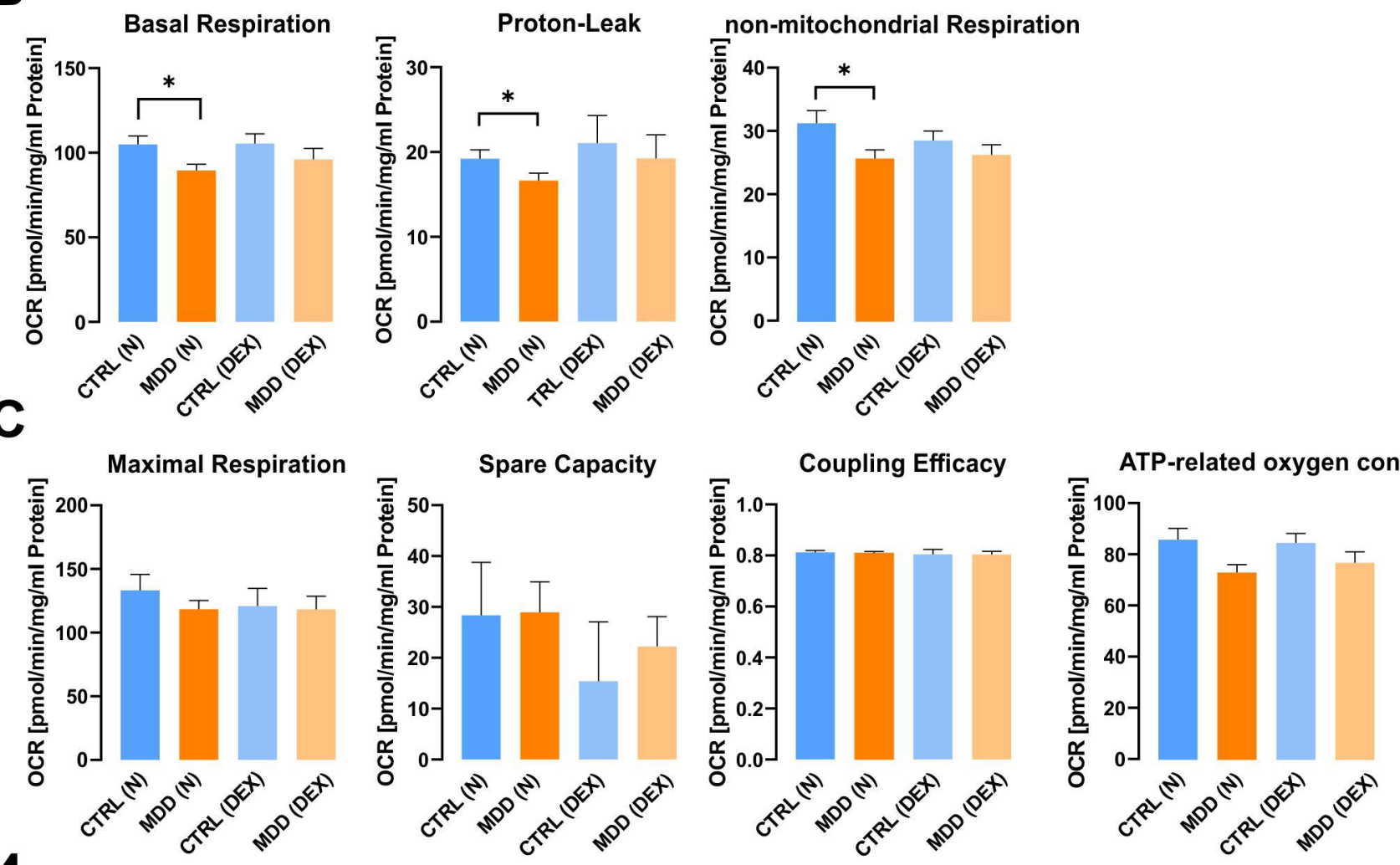

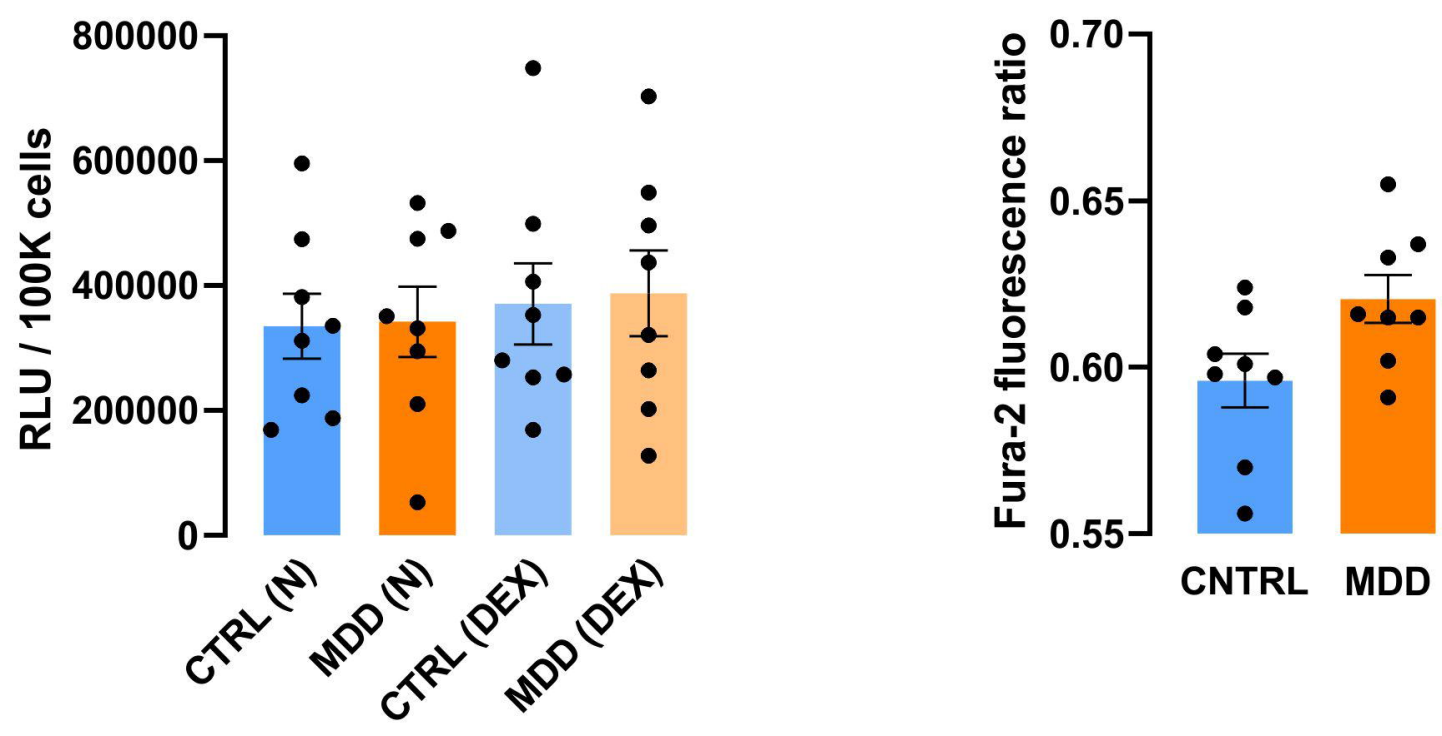

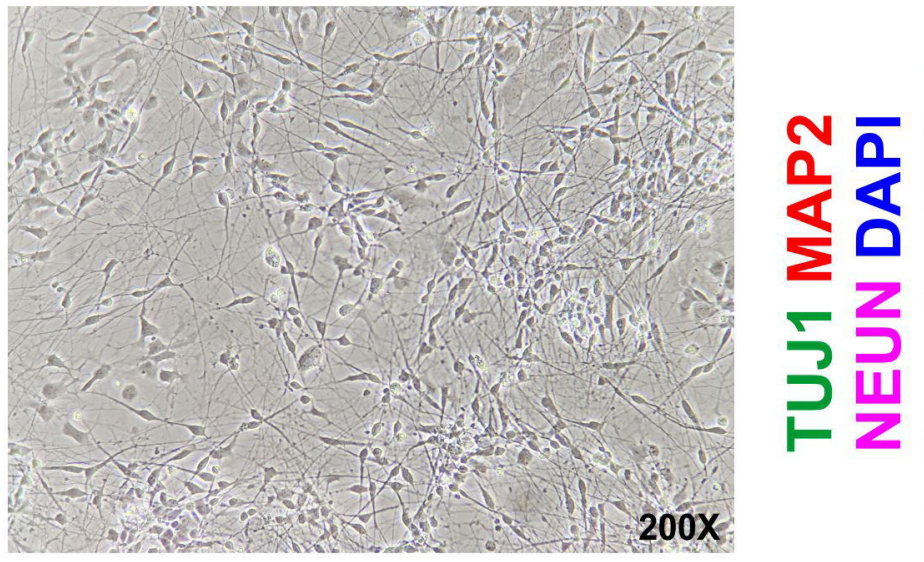

C
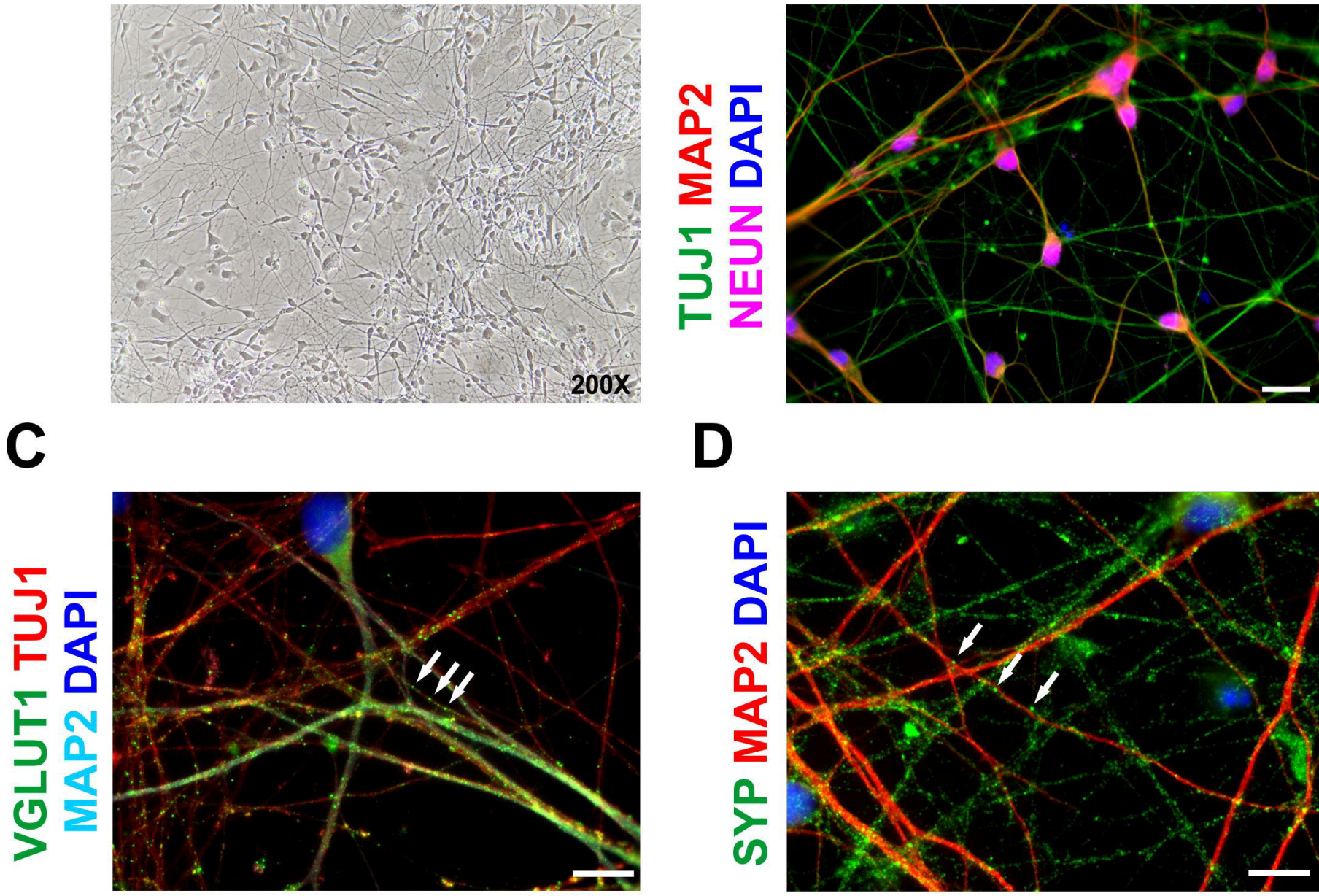

D

\section{Fig 6.}

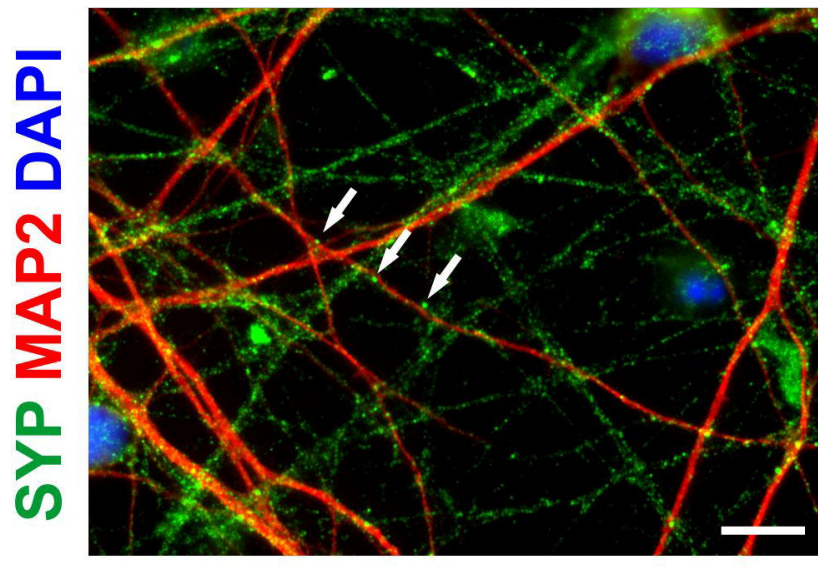



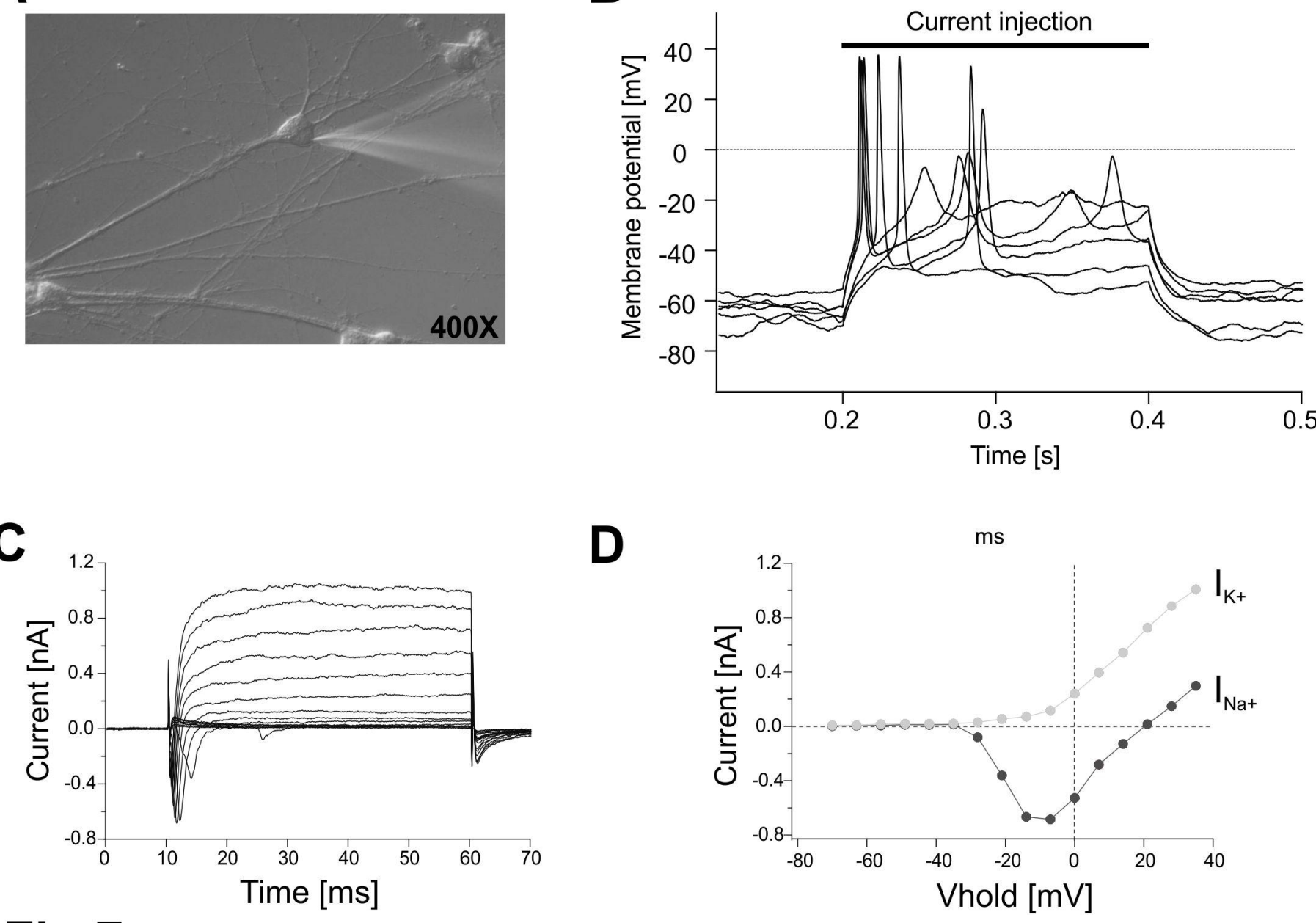

Fig 7. 


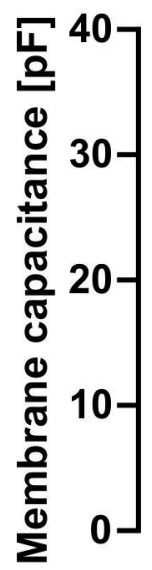

CNTRL

MDD

CNTRL

MDD
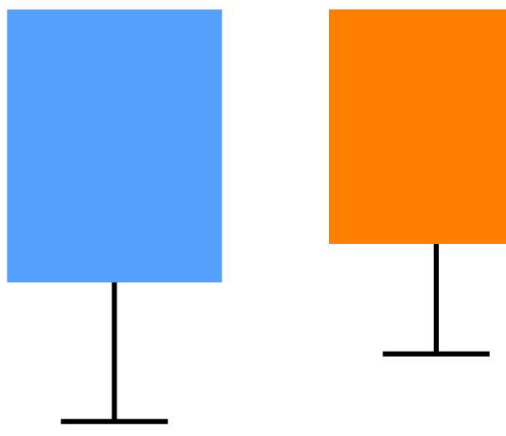


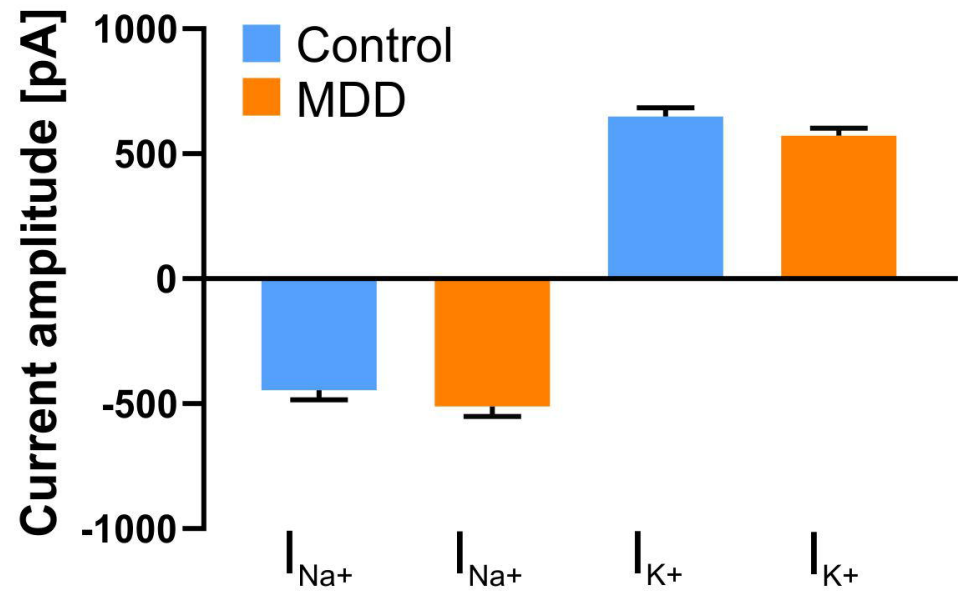

Spontaneous activity *

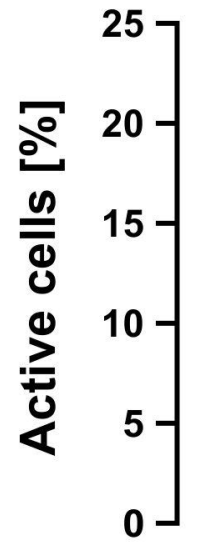

CNTRL MDD 